\title{
Design configuration of encoder and decoder modules for modified double weight (MDW) code spectral amplitude coding (SAC) optical code division multiple access (OCDMA) based on fiber Bragg gratings
}

\begin{abstract}
In this work, we are proposing the serial and parallel configurations of encoder and decoder modules to encode and decode a new developed spectral amplitude coding (SAC) known as modified double weight (MDW) code for optical code division multiple access (OCDMA) system. This coding scheme is designed in a way to decrease the number of FBGs used in the encoder and decoder modules and to maintain the cross-correlation parameter to 1 .
\end{abstract}

Keyword: Fiber Bragg grating; AND-subtraction scheme; Modified double weight code; Optical code division multiple access; Spectral amplitude coding 